\title{
O BALANCED SCORECARD COMO FERRAMENTA PARA IMPLANTAÇÃO DA ESTRATÉGIA: UMA PROPOSTA DE IMPLANTAÇÃO
}

\section{BALANCED SCORECARD AS TOOL FOR IMPLANTATION OF THE STRATEGY: AN IMPLANTATION PROPOSAL}

\author{
Odair Mesquita Quintella ${ }^{1}$; Gilson Brito Alves Lima ${ }^{2}$ \\ ${ }^{2}$ Universidade Federal Fluminense - UFF -Niterói - Brasil - quintela@uninet.com.br \\ ${ }^{2}$ Universidade Federal Fluminense - UFF - Niterói - Brasil - gilson@latec.uff.br \\ Recebido para publicação em: 16/07/05 \\ Aceito para publicação em: 14/06/05
}

\begin{abstract}
Resumo
Este trabalho versa sobre a implantação de um sistema de medição do desempenho, com base no balanced scorecard, que é uma ferramenta que permite traduzir a estratégia das organizações em objetivos e indicadores, por meio de perspectivas estratégicas, facilitando a divulgação $e$ disseminação das intenções da alta direção com todos os envolvidos. Apresenta dois modelos de aplicação do sistema de medição de desempenho. O primeiro deles, o de Kaplan e Norton e o segundo da Fundação para o Prêmio Nacional da Qualidade, comentando as características de cada um deles e as considerações a serem feitas quando da aplicação pelas organizações. É sugerido um modelo de implantação, com detalhamento das etapas a serem seguidas.
\end{abstract}

Palavras-chave: medição de desempenho; indicadores de desempenho; balanced scorecard.

\section{Introdução}

A avaliação do desempenho de uma organização deixou de ser apenas observado pelos resultados financeiros, que não são suficientes para garantir que suas estratégias, objetivos e metas estão sendo alcançados de formas sistêmica e global.

Segundo Lobato et al. (2003), da escola do planejamento financeiro dos anos 50, em que "... boa parte daquilo que se chamava planejamento financeiro era, na realidade, controle financeiro", passou-se, nos anos 90, para a gestão estratégica, em que o pensamento estratégico é contemplado e no qual há a integração entre planejamento e controle.

Os resultados da organização já não são mais apenas aqueles financeiros da escola dos anos 50. Eles passaram a ter uma conexão complexa entre as várias partes interessadas que estão relacionadas à organização: clientes, sociedade, acionistas, pessoas da sua força de trabalho e fornecedores.

Cada um desses grupos, que compõem as partes interessadas, tem interesses diferenciados que mudam de empresa para empresa e até mesmo, com o passar do tempo, para uma mesma empresa.

O quadro 1 apresenta um exemplo de meios de satisfazer as necessidades das partes interessadas de uma organização.

Quadro 1 - Exemplos de meios de satisfazer as partes interessadas 


\begin{tabular}{|l|l|l|l|l|}
\hline \multicolumn{1}{|c|}{ Clientes } & \multicolumn{1}{c|}{ Pessoas } & \multicolumn{1}{c|}{ Acionistas } & \multicolumn{1}{c|}{ Fornecedores } & \multicolumn{1}{c|}{ Sociedade } \\
\hline - preço & - moral & - dividendos & - parceria & - preservação do meio \\
- qualidade & - higiene e segurança & - valorização do & - volume de & ambiente \\
- variedade de & do trabalho & patrimônio & transações & - recolhimento de \\
produtos & - salários & & - preço de compra & impostos \\
- rapidez de entrega & - crescimento pessoal & & & \\
- inovação dos & e profissional & & & \\
produtos & & & & \\
\hline
\end{tabular}

Fonte: (Adaptado de Martins \& Costa Neto, 1998)

Para a organização, o processo de identificação das necessidades das partes interessadas é fundamental como base para definição de suas estratégias e planos que, por sua vez, irão definir as práticas de gestão e os processos operacionais que irão atender àquelas necessidades das partes interessadas.

Por sua vez, a definição de um sistema de indicadores de desempenho vem a ser a ferramenta que permite traduzir a estratégia da empresa em objetivos e metas.

Em 1992, o balanced scorecard - BSC - foi apresentado como sendo o sistema de medição do desempenho das organizações (Kaplan \& Norton, 1992) e desde então vem sendo usado cada vez mais por um número maior de organizações. No Brasil, pelo menos nos últimos cinco anos, as organizações reconhecidas pelo Prêmio Nacional da Qualidade - PNQ, estruturaram seu sistema de medição de acordo com um BSC ou uma forma adaptada, como mostrado a seguir

A Caterpillar (FPNQ, 2002a), premiada em 1999, utiliza um sistema, onde os indicadores são classificados em seis perspectivas e a Cetrel (FPNQ, 2002a), a segunda premiada, utiliza um sistema chamado "diagrama do sistema de indicadores - balanced scorecard".

Em 2001, a premiada no PNQ foi a Bahia Sul, que tem "o sistema de medição do desempenho ancorado no modelo Kaplan do Balanced Scorecard” (FPNQ, 2001).

Em 2002, foram três organizações premiadas. A Santa Casa da Misericórdia de Porto Alegre, uma delas, possui um sistema de medição no qual diversas correlações são consideradas (FPNQ, 2002b). Outra premiada, a Gerdau Aços Finos Piratini, relata como um exemplo de melhoria no sistema de medição do desempenho a "... nova forma de apresentação do desempenho global inspirada no modelo balanced scorecard ..." (FPNQ, 2002c) e a terceira, a Politeno, descreve que, em 2000, o sistema de indicadores passou por um redesenho utilizando a metodologia BSC - balanced scorecard. (FPNQ, 2002d).

Em 2003, a premiada com o PNQ foi o Escritório de Engenharia Joal Teitelbaum que relata ser "o sistema de medição do desempenho baseado na metodologia do balanced scorecard" (FPNQ, 2004)

Também no meio acadêmico-científico é grande o interesse pelo tema BSC. No XXIII Encontro Nacional de Engenharia de Produção - ENEGEP, cerca de 90\% dos trabalhos apresentados na área de medições de desempenho/gerência de produção tratavam do assunto.

\section{O modelo de Kaplan \& Norton}

O modelo de Kaplan e Norton - o balanced scorecard ou, simplificadamente, o BSC - é uma ferramenta que traduz a missão e a estratégia das empresas num conjunto abrangente de medidas de desempenho que serve de base para um sistema de medição e gestão estratégica e é organizado em torno de quatro perspectivas distintas - financeira, dos clientes, dos processos internos e do aprendizado e crescimento. O nome adotado procurava refletir o equilíbrio (balance) entre objetivos de curto e longo prazos, entre medidas financeiras e não-financeiras, entre indicadores de tendências e ocorrências e entre as perspectivas interna e externa de desempenho (KAPLAN e NORTON, 1997).

Na proposição desses autores, o estabelecimento da estratégia não é um processo gerencial isolado. É parte de um contínuo que tem seu início com a definição da missão da organização. É quando se tem a oportunidade de responder à pergunta "por que existimos?". A missão é a razão de 
ser da organização, a função que ela desempenha no mercado para tornar-se útil e justificar os seus resultados perante às partes interessadas.

Para se traduzir a missão em resultados almejados, percorre-se a trajetória que passa pelos valores essenciais - aquilo em que a organização acredita -, passa pela visão - o que se quer ser no futuro -, passa pela definição e implementação do sistema de medição - o BSC -, passa pelo estabelecimento das iniciativas estratégicas - o que é preciso ser feito - e se chegando ao nível pessoal - a contribuição de cada um para o alcance dos objetivos estratégicos.

Figura 1 - Traduzindo a missão em resultados almejados

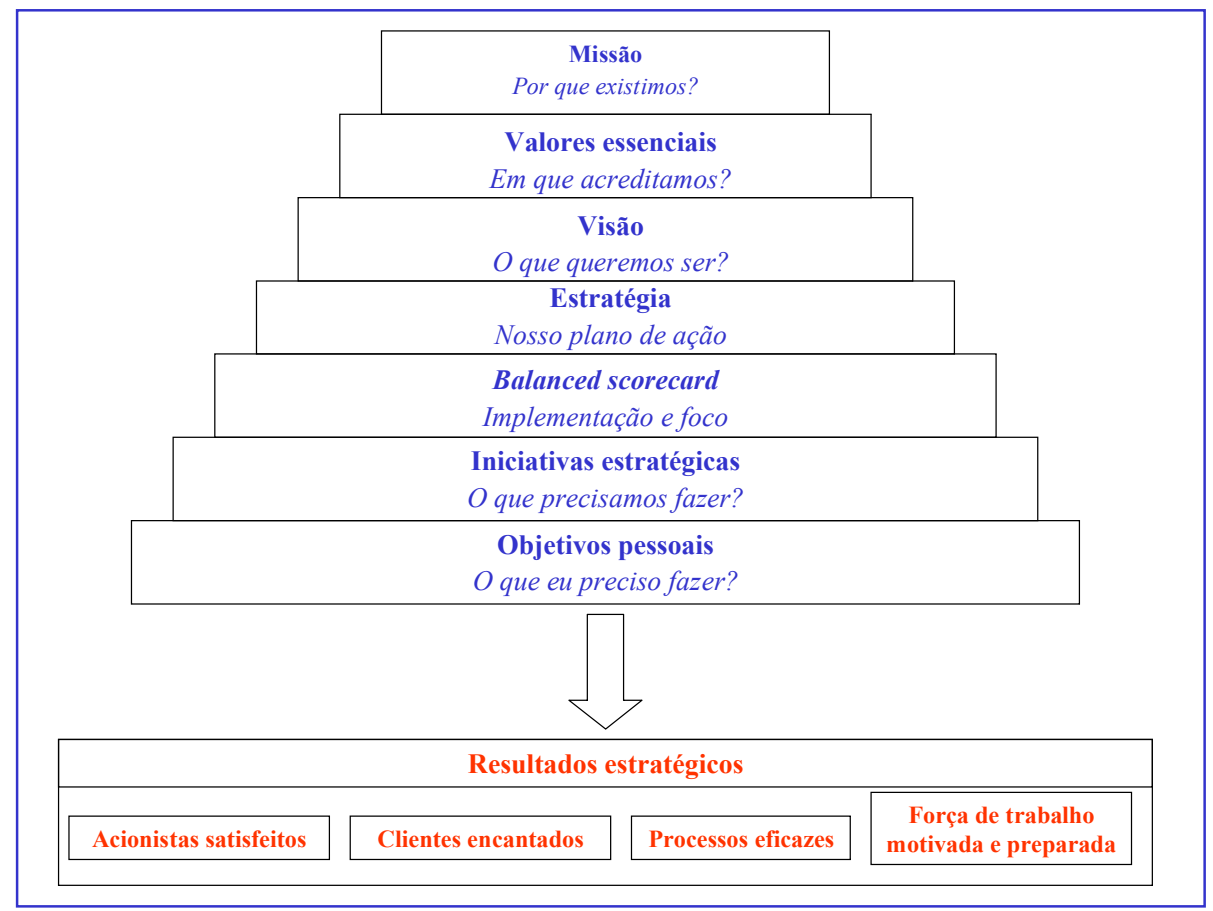

Fonte: (Adaptado de Kaplan e Norton, 2000)

De acordo com Kaplan \& Norton (1997), “... o sistema de medição deve tornar explícitas as relações [...] entre os objetivos (e as medidas) nas várias perspectivas, para que elas possam ser gerenciadas e validadas. A cadeia de causa e efeito deve permear todas as quatro perspectivas de um balanced scorecard". Essa cadeia de causa e efeito garantirá um encadeamento entre os objetivos das perspectivas, de modo a refletir as relações de causa e efeito assumidas na formulação das estratégias.

Quando da proposição inicial do balanced scorecard, em 1992, Kaplan \& Norton abordaram discretamente a forma de relacionamento entre as perspectivas e os respectivos objetivos delas decorrentes. Como os próprios autores afirmam, “... os scorecards estratégicos [foram construídos] como que a partir de uma folha de papel em branco". Posteriormente, como uma aplicação dos conceitos iniciais, e com base em centenas de scorecards desenvolvidos, aqueles autores propuseram um modelo chamado mapa estratégico, aonde os padrões da implantação vieram compor a referência para que a hipótese da estratégia fosse explicitada. Cada indicador do BSC se converte em parte integrante de uma cadeia lógica de causa e efeito que conecta os resultados almejados da estratégia com os vetores que induzirão a essas conseqüências. Em suma, os mapas estratégicos fornecem a base para a construção do BSC, estando este devidamente alinhado com a estratégia da organização (KAPLAN e NORTON, 1996a). 
Os mapas estratégicos ajudam as organizações a ver suas estratégias de maneira coesa, integrada e sistemática (figura 2).

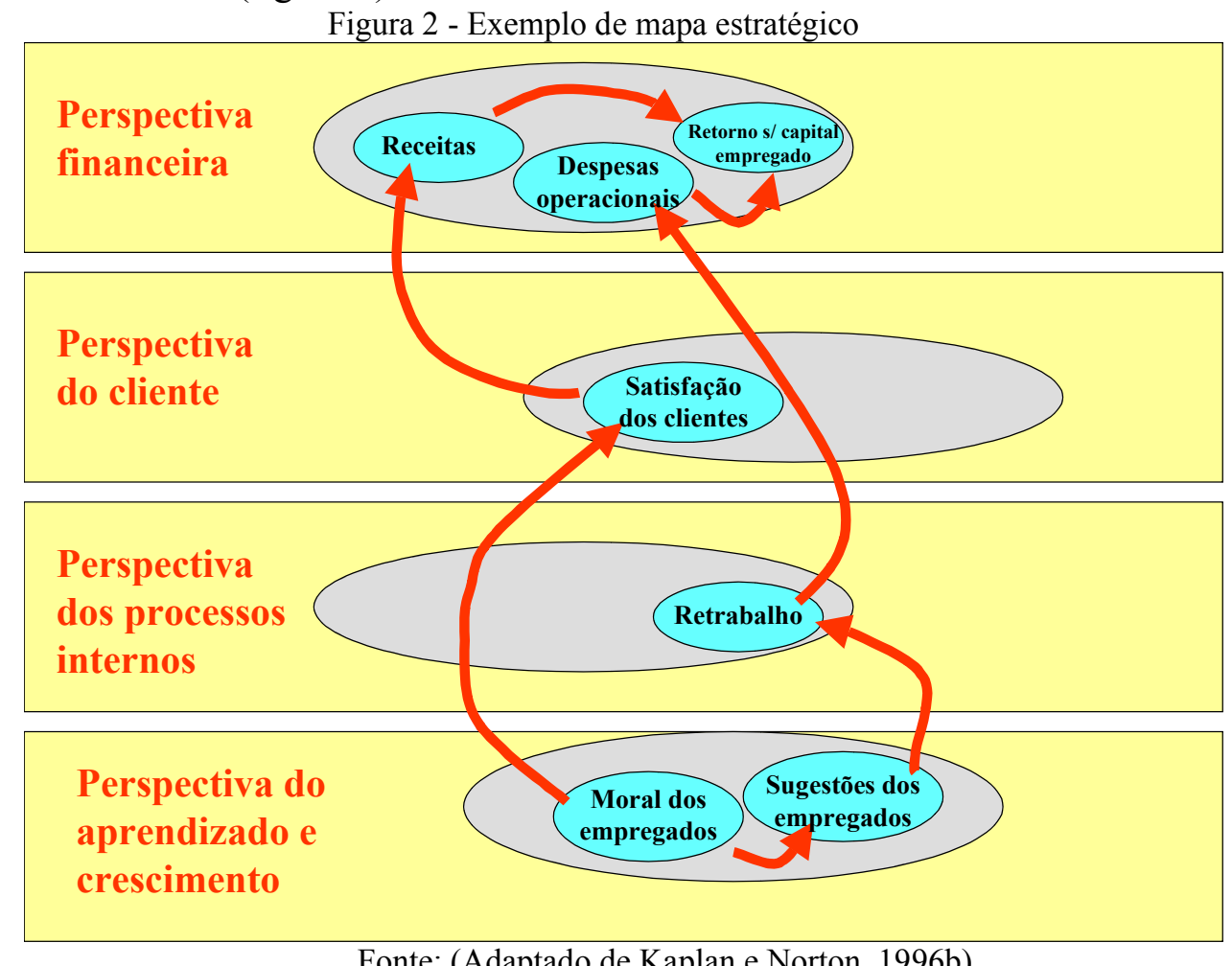

Fonte: (Adaptado de Kaplan e Norton, 1996b)

\section{O modelo da fundação para o prêmio nacional da qualidade}

À semelhança de David Norton e Robert Kaplan, que conduziram em 1990 reuniões bimestrais entre representantes de empresas de manufatura e serviços, da indústria pesada e de alta tecnologia para desenvolver um novo modelo de medição de desempenho, a Fundação para o Prêmio Nacional da Qualidade - FPNQ formou em agosto de 1999 um Comitê Temático, congregando representantes de cerca de 30 organizações dos mais diversos setores para discutir o tema "Medição do Desempenho Global".

Ambos trabalhos foram organizados em torno de perspectivas do negócio. A principal diferença entre os dois estudos está no número de perspectivas: o balanced scorecard de Kaplan e Norton considera as quatro perspectivas citadas anteriormente enquanto que o Comitê Temático da FPNQ considera oito - financeira, mercado/clientes, responsabilidade pública, inovação, processos, aquisição/fornecedores, pessoas e ambiente organizacional.

Um ponto de convergência encontrado entre os dois métodos - americano e brasileiro - é a utilização do mapa estratégico. No modelo da FPNQ, (2002a) há a recomendação de que o mapa chamado de diagrama de desdobramento da estratégia - “... deve incluir os principais desafios, obstáculos ou restrições ao atingimento dos objetivos e que resultam nos planos de ação, iniciativas, projetos etc.". O Relatório denomina de fatores críticos de sucesso os "desafios, obstáculos, restrições".

Os dois modelos também convergem quanto à importância de se ter um alinhamento entre o sistema de medição e a estratégia da organização.

A clareza na apresentação do diagrama permite uma visualização melhor dos objetivos e indicadores, uma análise mais objetiva das relações de causa e efeito e, principalmente, permite uma comunicação clara a todos na organização.

\section{O modelo proposto}


Para a implantação de um sistema de medição do desempenho, que reflita as estratégias da organização, é preciso observar certas etapas para que se tenha uma maior certeza de obtenção de sucesso nessa implantação.

O modelo proposto se baseia em exemplos reais de implantação e segue o esquema apresentado na figura 3 , que compreende as seguintes etapas:

- identificação das partes interessadas e de suas necessidades;

- diretrizes e valores organizacionais;

- análise estratégica;

- definição das estratégias;

- definição dos planos de ação; e

- sistema de medição - indicadores.

Figura 3 - Modelo de desenvolvimento de um sistema de medição de desempenho

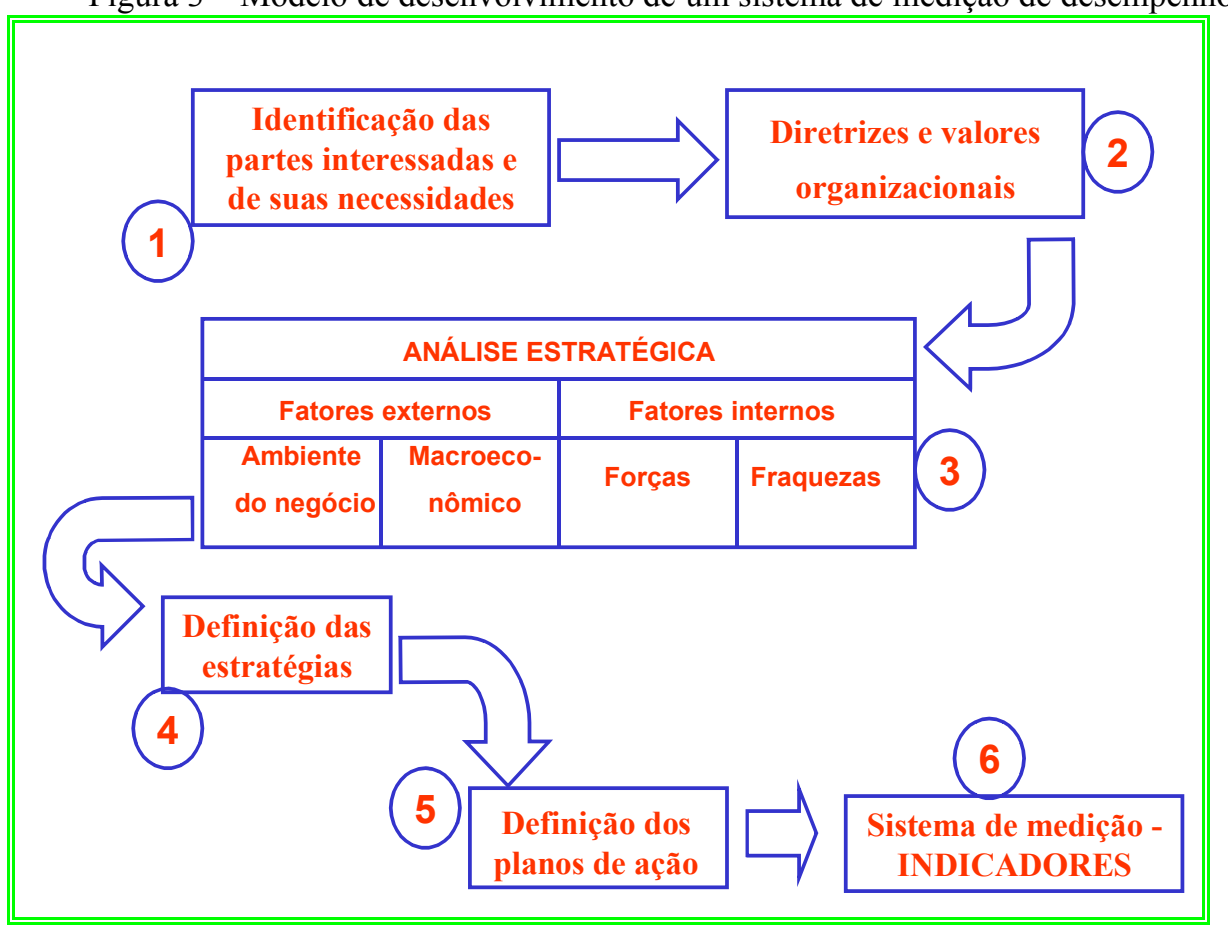

\subsection{Identificação das partes interessadas e de suas necessidades}

Esta etapa corresponde a um diagnóstico da estrutura da organização onde se procura definir o seu perfil, identificando quais são as partes interessadas e suas necessidades. Também são 
evidenciados os aspectos mais importantes dos processos de transformação dos insumos em produtos com valor agregado. Nesta etapa também são identificados aspectos relativos ao mercado em que a organização se situa e seus principais desafios.

O cuidado no detalhamento do perfil facilita as definições da missão, visão e valores organizacionais, como também na definição das estratégias.

Um exemplo da forma de detalhar o perfil pode ser encontrado em FPNQ, 2004b.

\subsection{Diretrizes e valores organizacionais}

Após a descrição do perfil da organização, quando as pessoas, e principalmente a alta direção, tiveram a oportunidade de fazer uma reflexão sobre os aspectos mais importantes dos seus processos - de produção, de apoio e de relacionamento com todas as partes interessadas - a organização define suas diretrizes e seus valores. $\mathrm{O}$ autoconhecimento é um importante produto da discussão e descrição do perfil.

É comum as organizações envolverem diversos níveis na discussão dos seus valores e diretrizes, e com isso buscar obter o envolvimento do maior número possível de pessoas e diminuir os riscos de um insucesso no alcance dos objetivos, mas a responsabilidade, em última instância, pela sua definição é da alta direção.

\subsubsection{Missão}

O processo de elaboração da missão se torna simples se alguns pontos forem observados. Esses pontos podem ser definidos ao se responder questões/itens relacionados a atividade, produtos, mercados, identidade, ênfase e outros aspectos considerados relevantes.

\subsubsection{Visão}

Para a definição da visão devem ser considerados aspectos relacionados ao estado que a organização deseja atingir no futuro (FPNQ, 2004a).

Como forma prática de sua definição, precisam ser respondidas perguntas do tipo: "o que a organização será?"; "o que a organização fará?"; “como a organização estará?"; e “onde a organização estará?”.

Sendo a visão uma imagem do que a organização quer criar no futuro, é importante não perder de vista que ela parte do presente. Assim, é fundamental que a missão esteja muito clara para toda a organização.

\subsubsection{Valores}

Os valores, ou valores organizacionais, são os "entendimentos e expectativas que descrevem como os profissionais da organização se comportam e sobre os quais todas relações organizacionais estão baseadas" (FPNQ, 2004a).

Os valores fazem parte da cultura organizacional. No entanto é da maior importância ter em mente que, por mais que a organização procure oferecer condições de participação para as pessoas, o grupo de comando é quem determina o que a empresa quer ser e o que fará. Isto faz com que os valores da organização, e conseqüentemente a sua cultura, tenham muito (ou quase tudo) da alta direção.

Os valores representam o que a organização dá maior importância. Como devem ser observados pelas pessoas, é importante que sejam entendidos e aceitos por elas. 


\subsection{Análise estratégica}

A definição das estratégias da organização tem por base a análise dos fatores que impactam nos seus resultados. Essa análise é feita com base nos cenários em que a organização se situa. Devem ser considerados os fatores externos - fatores do ambiente do negócio e fatores macroeconômicos - e fatores internos - relacionados às funções existentes na organização.

A análise dos fatores externos permite identificar as ameaças e oportunidades do ambiente em que a organização se posiciona e definir as estratégias para neutralizar as ameaças e potencializar as oportunidades.

Quando da análise dos fatores internos, são considerados os pontos fortes e fracos, relacionados às funções existentes na organização. São aqueles controláveis pela organização.

\subsection{Definição das estratégias}

O presente modelo proposto considera os mapas estratégicos propostos por Kaplan \& Norton e pelo modelo da Fundação para o Prêmio Nacional da Qualidade.

Inicialmente é preciso identificar as perspectivas relacionadas ao negócio da organização. O sucesso na definição dessas perspectivas é tão maior quanto o cuidado que se teve na definição dos itens anteriores: perfil, missão, visão, valores e análise estratégica.

Com as perspectivas definidas, o passo seguinte é desenhar a forma de seu relacionamento o mapa estratégico - onde as mesmas guardam uma relação de causa e efeito.

A definição de um mapa que espelhe a realidade da organização facilita a posterior definição dos indicadores de forma equilibrada.

\subsection{Definição dos planos de ação}

Para cada perspectiva definida no item anterior, a organização define pelo menos um objetivo estratégico.

Os objetivos devem guardar a mesma relação das perspectivas. Isto significa que um objetivo está diretamente relacionado a outro.

Para cada objetivo então estabelecido são identificados os desafios, obstáculos ou restrições que se apresentam e que podem dificultar o atingimento do objetivo.

O conjunto desses desafios, obstáculos ou restrições permite estabelecer os planos de ação que são detalhados para mostrar o caminho a ser seguido e com isso se possa alcançar os objetivos traçados.

\subsection{Sistema de medição - indicadores}

Respeitadas as etapas até agora descritas, o passo seguinte é o estabelecimento dos indicadores que apoiam a implantação das estratégias e permitem o acompanhamento do progresso dos planos de ação estabelecidos.

O sistema de medição então criado é estruturado de tal forma que facilite a análise critica dos resultados alcançados, relativos aos objetivos e planos de ação traçados, e permita a tomada de decisões com maior segurança e facilidade.

\section{Conclusões}

A observância de um caminho a seguir na implantação de um sistema de indicadores de desempenho aumenta a probabilidade de sucesso nesse processo de se estabelecer as estratégias.

Ao se adotar o modelo da Fundação do Prêmio Nacional da Qualidade, passa-se a ter a opção de oito perspectivas, o que permite uma definição mais clara das estratégias da organização e, conseqüentemente, uma relação mais apropriada dos indicadores. 
O modelo proposto procura trazer uma contribuição para o processo de definição do sistema de indicadores em que seja respeitado o caminho que começa com a discussão do perfil da organização, passando pelas diretrizes organizacionais, objetivos estratégicos e correspondentes planos de ação, que permite, de forma pragmática, atingir a sua missão e a sua visão.

\begin{abstract}
This paper investigates the implementation of performance measurement system, based on the balanced scorecard, which is a tool that allows to translate the strategy of the organizations in objectives and indicators, by using strategic perspectives, facilitating the widespread and dissemination of the intentions of the board with the whole team. It also presents two models of application of the performance measurement system. The first, is the Kaplan and Norton's and the one proposed by the Brazilian Foundation for the National Quality Award (FPNQ), describing the characteristics of each of them and the considerations to be done when applied by different organizations. It suggests an implantation model, with the detailed steps to be followed.
\end{abstract}

Key-words: Performance measurement, performance indicators, balanced scorecard.

\title{
Referências
}

FUNDAÇÃO PARA O PRÊMIO NACIONAL DA QUALIDADE. Relatório da Gestão - Bahia Sul Celulose S.A. São Paulo: FPNQ, 2001.

Fundação para o Prêmio Nacional da Qualidade. Caso para Estudo - Escritório de Engenharia Joal Teitelbaum. São Paulo: FPNQ, 2004b.

Fundação para o Prêmio Nacional da Qualidade. Relatório da Gestão da Gerdau Aços Finos Piratini S.A. São Paulo: FPNQ, 2002c.

Fundação para o Prêmio Nacional da Qualidade. Critérios de excelência 2004 - O estado da arte da gestão para a excelência do desempenho e o aumento da competitividade. Rev. 1. São Paulo: FPNQ, 2004a.

Fundação para o Prêmio Nacional da Qualidade. Relatório da Gestão da Irmandade Santa Casa de Misericórdia de Porto Alegre. São Paulo: FPNQ, 2002b.

Fundação para o Prêmio Nacional da Qualidade. Relatório da Gestão da Politeno. São Paulo: FPNQ, 2002 d.

Fundação para o Prêmio Nacional da Qualidade. Planejamento do sistema de medição do desempenho. 2. ed. São Paulo: FPNQ, 2002a.

KAPLAN, Robert S.; NORTON, David. P. Using the balanced scorecard as a strategic management system. Harvard Business Review, Boston, v. 74, n. 1, p. 75-85, Jan./Feb. 1996b.

KAPLAN, Robert S.; NORTON, David P. A estratégia em ação - balanced scorecard. 13. ed. Rio de Janeiro: Campus, 1997.

KAPLAN, Robert S.; NORTON, David P. Linking the balanced scorecard to strategy. California Management Review, Berkeley, v. vol. 39, n. 1, p. 53-79, out. 1996a.

KAPLAN, Robert S.; NORTON, David P. The balanced scorecard - measures that drive performance. Harvard Business Review, Boston, v. 70, p. 71-79, Jan./Feb. 1992.

KAPLAN, Robert S.; NORTON, David P. Organização orientada para a estratégia: como as empresas que adotam o balanced scorecard prosperam no novo ambiente de negócios. Rio de Janeiro: Campus, 2000.

LOBATO, David Menezes et al. Estratégia de empresas. 2. ed. Rio de Janeiro: FGV, 2003.

MARTINS, Roberto Antonio; COSTA NETO, Pedro Luiz de Oliveira. Indicadores de desempenho para a gestão pela qualidade total: uma proposta de sistematização. Gestão \& Produção, São Carlos, v. 5, n. 3, p. 298-311, dez. 1998. 